\title{
Simple Waves Do Not Avoid Eigenvalue Crossings
}

\author{
LYUBA CHUMAKOVA \\ Courant Institute \\ ESTEBAN G. TABAK \\ Courant Institute
}

\begin{abstract}
General one-parameter families of matrices avoid eigenvalue crossings. It is shown that the matrices associated with the simple waves of nonlinear systems of conservation laws do not obey this rule: a subset of simple waves with nonzero measure has crossing eigenvalues. (C) 2009 Wiley Periodicals, Inc.
\end{abstract}

\section{Introduction: Avoidance of Crossing}

During the early days of quantum mechanics, physicists discovered that an $n \times n$ matrix $A(t)$, depending on a parameter $t \in R$, is not likely to have multiple eigenvalues for any value of $t[6,8]$. For example, consider the one-parameter family of matrices $A(t)=B+t M$, where $B$ and $M$ are two real $n \times n$ symmetric matrices chosen at random. The $n$ eigenvalues are all real and depend on $t$. Typically (see Figure 1.1 as an example), as $t$ approaches a certain value $t^{*}$, the eigenvalues seem to be about to collide; yet they suddenly turn away from each other and avoid crossing. This was first explained by Wigner and von Neumann; the explanation hinges on the size of the set of real, symmetric matrices with multiple eigenvalues, called degenerate in the physics literature. The dimension of the space of real $n \times n$ symmetric matrices is $N=\frac{n(n+1)}{2}$, while the dimension of its subspace with repeated eigenvalues is $N-2$. In general, a one-dimensional curve $A(t)$ will not intersect a codimension 2 manifold. Thus it is said that the eigenvalues avoid crossings.

One can verify the dimensionalities quoted above by computing the number of parameters needed to determine a matrix completely. In the case of real symmetric matrices, one needs first $n$ parameters to specify the $n$ eigenvalues. Specifying the corresponding eigenvectors requires $n-1$ parameters for the first, since the vector's norm is immaterial, $n-2$ for the second, since it is orthogonal to the first, and so on, all the way down to one parameter for the $(n-1)^{\text {th }}$, since the $n^{\text {th }}$ eigenvector is fully determined by its orthogonality to all the others. The total number of parameters needed adds up to $N=\frac{n(n+1)}{2}$.

If the matrix has repeated eigenvalues, on the other hand, one needs $n-1$ parameters to specify the eigenvalues, then $n-1$ for the first eigenvector, and so on 


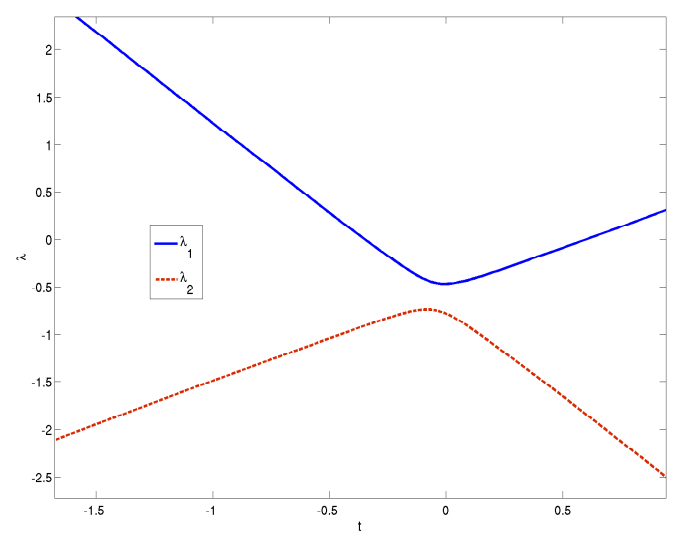

FiguRE 1.1. Eigenvalues of a matrix $A+t B$ do not cross as $t$ changes. Here $A$ and $B$ are random symmetric $2 \times 2$ matrices.

as before, but only down to 2 for the $(n-2)^{\text {th }}$ eigenvector: the last two eigenvectors, corresponding to the repeated eigenvalue, are any pair that spans the twodimensional subspace orthogonal to the first $n-2$. The total number of parameters needed becomes $N-2$; there is one less eigenvalue and one less eigenvector to specify.

We discovered that, if the one-dimensional curve $A(t)$ is chosen to correspond to the phase space representation of a simple wave, the crossing of eigenvalues is not exceptional, unlike in the general case. The points where the matrix is degenerate are attractors for the simple-wave dynamics in phase space. This paper is devoted to understanding this curious phenomenon.

\subsection{Simple Waves}

Consider a linear system of hyperbolic PDEs with constant coefficients, encapsulated in a matrix $A$ :

$$
u_{t}+A u_{x}=0, \quad u \in \mathbb{R}^{n}, x, t \in \mathbb{R}, u=u(x, t) .
$$

It is well-known that, when $A$ has real eigenvalues and a complete set of eigenvectors, the solution $u$ can be decomposed into $n$ waves, or "modes of oscillation," of the system. One way to describe these modes is by considering solutions with only one degree of freedom: $u(x, t)=U(\theta(x, t))$. Plugging this ansatz into (1.1), one obtains the eigenvalue problem $(A-\lambda I) U_{\theta}=0$ and the advection equation for $\theta: \theta_{t}+\lambda \theta_{x}=0$. Since the matrix $A$ is constant, the eigenvalues and eigenvectors are also constant. Therefore, all the $n$ solutions $U_{j}(\theta)$ are proportional to the corresponding eigenvector $w_{j}$ :

$$
U_{j}(\theta)=U_{j}^{0}+\theta_{j} w_{j}
$$


and propagate along straight characteristics, at a speed given by the corresponding eigenvalue:

$$
\begin{aligned}
& \theta_{j}(x, t)=\theta_{j}^{0}\left(x-\lambda_{j} t\right) \quad \text { where } \theta_{j}^{0}(x)=\theta_{j}(x, 0), \\
& u_{j}(x, t)=U_{j}(\theta)=U_{j}^{0}+\theta_{j}^{0}\left(x-\lambda_{j} t\right) w_{j} .
\end{aligned}
$$

In the more general case of quasi-linear systems, the matrix $A$ depends on $u$. The analogue of the linear modes of oscillation are the simple waves, which are fully nonlinear solutions to the system. Unlike the linear modes, the simple waves can deform and break. Also, since they are solutions of a nonlinear system, they only exist in isolation: a superposition of simple waves would interact and hence not be "simple" anymore.

For a nonlinear hyperbolic system of the type

$$
u_{t}+A(u) u_{x}=0, \quad u \in \mathbb{R}^{n}, t, x \in \mathbb{R},
$$

where $A$ is an $n \times n$ real matrix, simple waves are solutions of the form

$$
u(x, t)=U(\theta(x, t))
$$

all components of the vector solution $u$ are functions of a single scalar function $\theta(x, t)$. Plugging this ansatz into (1.4) and rearranging the terms, we obtain

$$
A(U) U_{\theta}=-\frac{\theta_{t}}{\theta_{x}} U_{\theta},
$$

which yields the eigenvalue problem

$$
[A(U(\theta))-\lambda(U(\theta)) I] U_{\theta}=0,
$$

where $\theta(x, t)$ is described by the evolution equation

$$
\theta_{t}+\lambda(U(\theta)) \theta_{x}=0
$$

The eigenvector $U_{\theta}$ in the eigenvalue problem (1.6) yields a system of ODEs for $U(\theta)$. We will refer to the function $U(\theta)$ as a phase space representation of the simple wave, as opposed to its evolution in the physical space of $x$ and $t$.

One can look at general solutions $u(x, t)$ as evolving curves in phase space $u \in \mathbb{R}^{n}$, each curve parametrized by $x$, and the curve's evolution by $t$. The simple waves constitute the special case of fixed curves $U(\theta)$ in phase space, which do not change in time (their parametrization changes though, since $\theta=\theta(x, t)$ ). Note that the choice of $\theta$ is not unique; it can be replaced by any function of $\theta$.

It was shown by Friedrichs [4] (see also $[3,5]$ ) that simple waves are the solutions of quasi-linear systems in the regions adjacent to uniform states $u(x, t)=$ const. Simple waves in physical space also arise as building blocks for the solution to Riemann problems and, more generally, in the response of quasi-linear systems to localized initial conditions or forcing events. 
In this paper, we study the dynamics of simple waves not in the $x t$-plane, but in the phase space $\mathbb{R}^{n}$, analyzing the system of ODEs

$$
\frac{d U}{d \theta}=w \quad \text { where }(A(U)-\lambda I) w=0 .
$$

We find that the points $U$ with double eigenvalues have attracting sectors for the simple wave trajectories $U(\theta)$.

In the following text we will frequently think of $\theta$ as a "time" along the simple wave trajectory. However, $\theta$ should not be confused with the time from the original PDE; the $x t$-evolution of $\theta$ is given by (1.7). Note that the right eigenvector $w$ is defined only up to a scalar factor, and that different choices for $w$ lead to a reparametrization of the time $\theta$ along a simple wave.

Often in the text that follows, we will need to say that the crossing of eigenvalues for simple waves is not exceptional, as it is for general one-parameter families of matrices. We can think of this "nonexceptionality" in probability terms, through a thought experiment in which we draw an initial condition for a simple wave at random from phase space, and show that, with nonzero probability, this simple wave leads to an eigenvalue crossing. When, later in the text, we speak of nonzero probabilities, the just-described thought experiment is implied.

\subsection{Double Eigenvalues and Wave Interaction}

The appearance of double eigenvalues in the system $u_{t}+A(u) u_{x}=0$ is not just a matter of curiosity. When the system considered is hyperbolic, the $n$ eigenvectors of $A(u)$ determine $n$ waves that travel at speeds given by the corresponding eigenvalues. Note that waves traveling at the same speed remain in phase with each other, and hence can interact in a fully nonlinear manner, while waves with compact support that travel at different speeds only "see" each other for a finite time interval. Thus the frequency of scenarios when a pair of eigenvalues collide allows one to derive conclusions about the nature and strength of the system's nonlinear wave interaction.

\subsection{Double Eigenvalues and Nonlinear Stability}

Double eigenvalues also impact the system's stability. Systems of conservation laws of the form (1.4) may be of mixed type, with real eigenvalues for some values of $u$, and at least some complex eigenvalues for others. The complex eigenvalues correspond to instabilities of the system, since arbitrarily small perturbations may grow explosively. In mathematical terms, this corresponds to ill-posedness, arising from considering the variable $t$ timelike in a system that is elliptic.

One can translate these thoughts into a local characterization of stability [1, 2, 7], describing the system as stable (well-posed) where it is hyperbolic, and unstable (ill-posed) where it is elliptic (i.e., some eigenvalues become complex). Since all the points on the boundary between the hyperbolic and elliptic domains (later referred to as the HE boundary) have repeated eigenvalues, studying such points helps us understand the system's stability properties. In particular, the question 
of whether such points are dynamically reachable from the hyperbolic side is a mathematical formulation of nonlinear stability.

We find that there are sectors of simple waves passing through the points yielding double eigenvalues. This suggests that, near the HE boundary, general solutions of (1.4) might be closely approximated by a simple wave. Note that the simple wave trajectories $U(\theta)$ are fixed in phase space for all times $t$, and hence are nonlinearly stable: if they start hyperbolic, they will remain so for all time up to breaking. Hence the fact that simple waves do not avoid crossing in phase space may inhibit the nonlinear transition to instability in systems of mixed type. (This statement requires a qualification though: only at exceptional points on the $\mathrm{HE}$ boundary is there a complete set of eigenvectors associated to the repeated eigenvalues, the situation considered in this paper.)

\subsection{Plan of the Paper}

First we study the phase space dynamics of simple waves in its simplest instance, where $A$ is a $2 \times 2$ matrix. In later sections we consider the general case, where $A$ is $n \times n$.

\section{Problem Setup}

Consider a quasi-linear system with a matrix $A(u)$ that depends on the vector $u(x, t) \in \mathbb{R}^{n}$, satisfying

$$
u_{t}+A(u) u_{x}=0
$$

We assume that the system is locally hyperbolic; i.e., we consider the domain where $A(u)$ has only real eigenvalues and a complete set of eigenvectors. In general, a solution to (2.1) can be viewed as forming a two-dimensional surface, parametrized by $t$ and $x$, in the $n$-dimensional phase space. We consider special solutions, the simple waves, which can be obtained by requiring the surface to degenerate into a curve, so that the range of the solution is one-dimensional. Such $u$ can be represented in the form $u=U(\theta)$, where the scalar $\theta$ is a function of $x$ and $t$. This yields

$$
U^{\prime}(\theta) \theta_{t}+A(U) U^{\prime}(\theta) \theta_{x}=0 .
$$

In this paper we will only consider the dynamics in phase space, where $u=U(\theta)$. Since we will not concern ourselves with the space-time evolution of $u$, we switch from the notation $u(x, t)$ to $u=u(\theta)$, standing for $u=U(\theta)$. Thus (2.2) becomes

$$
\frac{d u}{d \theta} \theta_{t}+A(\theta) \frac{d u}{d \theta} \theta_{x}=0,
$$

yielding an advection equation for the parameter $\theta$, and a system of ODEs for the simple wave $u(\theta)$ :

$$
\begin{gathered}
\theta_{t}+\lambda_{i}(\theta) \theta_{x}=0 \\
\text { where } \frac{d u}{d \theta}=w_{i}(\theta), A w_{i}(\theta)=\lambda_{i}(\theta) w_{i}(\theta), i \in 1,2, \ldots, n .
\end{gathered}
$$


After choosing an initial condition $u^{0}$ and a particular family $i$ of eigenvalues, we follow the corresponding eigenvector to obtain a simple wave in phase space. We are interested here in the case when the eigenvalues of the matrix $A(u)$ may cross but not form a Jordan block. In other words, $A(u)$ is degenerate yet diagonalizable. We call the values of $u$ yielding such crossings the $D D$ points.

\section{$2.12 \times 2$ Symmetric Case}

In the two-dimensional symmetric case, $\mathbf{u}=(u, v) \in \mathbb{R}^{2}$, and $A=A^{\top}=A(\mathbf{u})$. Let $\left(u_{0}, v_{0}\right)$ be a $D D$ point for the matrix $A(\mathbf{u})$, where the repeated eigenvalue is $\lambda \in \mathbb{R}$. Without loss of generality, we shift the point $\mathbf{u}_{0}=\left(u_{0}, v_{0}\right)$ to the origin, so that $(u, v)=(0,0)$ is a $D D$ point. Near the $D D$ point, we obtain to leading order

$$
A(\mathbf{u})=\lambda I+u A_{u}+v A_{v}=\left[\begin{array}{ll}
f_{1} & f_{2} \\
f_{2} & f_{4}
\end{array}\right],
$$

where $A_{u}$ and $A_{v}$ are constant $2 \times 2$ symmetric matrices and $I$ represents the identity. Then

$$
\left[\begin{array}{ll}
f_{1} & f_{2} \\
f_{2} & f_{4}
\end{array}\right]=\left[\begin{array}{cc}
\lambda+a_{1} u+b_{1} v & a_{2} u+b_{2} v \\
a_{2} u+b_{2} v & \lambda+a_{4} u+b_{4} v
\end{array}\right]
$$

for some constants $a_{1}, a_{2}, a_{4}, b_{1}, b_{2}$, and $b_{4}$. The system of ODEs along a simple wave becomes

$$
\frac{d u}{d v}=\frac{-1}{\frac{f_{1}-f_{4}}{2 f_{2}} \pm \sqrt{\left(\frac{f_{1}-f_{4}}{2 f_{2}}\right)^{2}+1}},
$$

where the right-hand side is the ratio of the components of the right eigenvector $w$. Note that the right-hand side is homogeneous in $u$ and $v$. Changing variables from $(u, v)$ to $(q, v)$, where $q=\frac{u}{v}$ is the cotangent of the polar angle, the system decouples:

$$
-\frac{d v}{v}=\frac{d q}{q-m \pm \sqrt{m^{2}+1}}
$$

where $m=\frac{f_{1}-f_{4}}{2 f_{2}}$ is a function of $q$ only. Hence, introducing a time parameter $\theta$ such that

$$
d \theta=-\frac{d v}{v}=\frac{d q}{q-m \pm \sqrt{m^{2}+1}},
$$

the simple waves are described by the system

$$
\left\{\begin{array}{l}
\frac{d v}{d \theta}=-v, \\
\frac{d q}{d \theta}=q-m \pm \sqrt{m^{2}+1}, \quad m=\frac{f_{1}-f_{4}}{2 f_{2}} .
\end{array}\right.
$$

Observe that the first equation has a stable fixed point at $v=0$. In the next subsection we consider the behavior of $q$ as $\theta \rightarrow \infty$. Since $u=q v$, and $v$ converges to 0 when $\theta \rightarrow \infty$, it is enough, in order for $u$ also to converge to 0 , that $q$ remain bounded. We will show that, with nonzero probability, the second equation in (2.4) has a stable fixed point $q^{*} \in \mathbb{R}$. Therefore, for all $q$ 's in the 


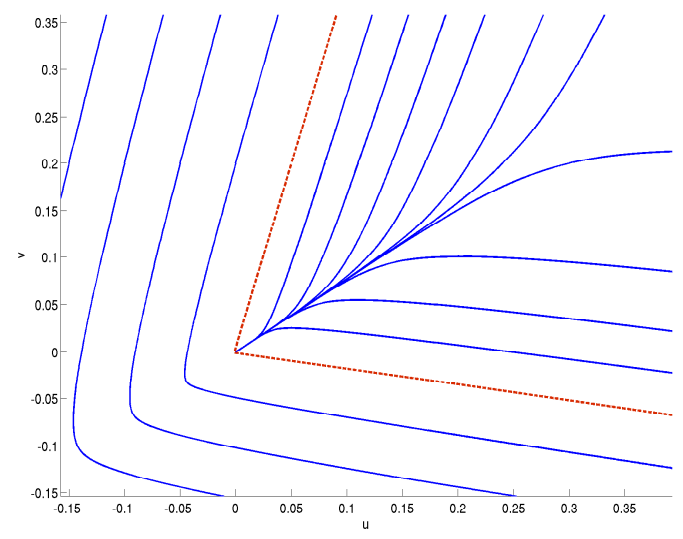

FIGURE 2.1. The $D D$ point $(u, v)=(0,0)$ has an attracting sector on the $(u, v)$ phase plane, marked by the dashed lines.

domain of attraction of $q^{*}$, the trajectories remain bounded for all times $\theta$, and $(u, v) \rightarrow(0,0)$. In other words, there is an interval for the quotient $\frac{u}{v}$ in the initial conditions for which the simple wave tends to the degenerate point.

\subsection{One-Dimensional Dynamics of $q(\theta)$}

Consider the dynamics of $q(\theta)$ :

$$
\begin{aligned}
\frac{d q}{d \theta}=f(q) & =q-\frac{f_{1}-f_{4}}{2 f_{2}} \pm \sqrt{\left(\frac{f_{1}-f_{4}}{2 f_{2}}\right)^{2}+1} \\
& =q-\frac{a_{3} q+b_{3}}{a_{2} q+b_{2}} \pm \sqrt{\left(\frac{a_{3} q+b_{3}}{a_{2} q+b_{2}}\right)^{2}+1},
\end{aligned}
$$

where $a_{3}=\left(a_{1}-a_{4}\right) / 2$ and $b_{3}=\left(b_{1}-b_{4}\right) / 2$.

To study this ODE, we analyze its fixed points and their stability. Depending on the three parameters $\left(a_{3} / a_{2}, b_{3} / a_{2}\right.$, and $\left.b_{2} / a_{2}\right), f(q)$ has the following properties: it increases on most of the real line, has a vertical asymptote at $q=-b_{2} / a_{2}$, and approaches $y=q$ asymptotically as $q \rightarrow \pm \infty$. Hence, with nonzero probability, the equation $\frac{d q}{d \theta}=f(q)$ will have a stable fixed point at some $q^{*} \in \mathbb{R}$. Because of the asymptotic behavior of $f(q)$ at $q \rightarrow \pm \infty$, the fixed point $q=q^{*}$ needs to have an unstable fixed point at each side. These unstable points constitute the boundaries of the sector in which all the trajectories converge to $(u, v)=(0,0)$ along the line $\frac{u}{v}=q^{*}$. We demonstrate a realization of this scenario in the example below and in Figure 2.1.

For the set of parameters

$$
\left[\begin{array}{ll}
a_{3} & b_{3} \\
a_{2} & b_{2}
\end{array}\right]=\left[\begin{array}{rr}
0.7544 & -0.9358 \\
-0.2361 & 0.4716
\end{array}\right]
$$


$f(q)$ has an unstable fixed point at $q=q_{1}$ and a stable fixed point at $q=q^{*}$. The asymptote is at $q_{2}=-b_{2} / a_{2}$. The plot on the right shows the corresponding $(u, v)$ phase portrait for the system (2.4). As expected, all the trajectories in the cone bounded by $\frac{u}{v}=q_{1}$ and $\frac{u}{v}=q_{2}$ converge to the trajectory $\frac{u}{v}=q^{*}$.

Note that the system has no distinguished scale, because the dynamics depends only on the ratio $\frac{u}{v}$. Therefore, the phase portrait shown in Figure 2.1 is representative of the phase portrait in all of $\mathbb{R}^{2}$ provided that $(u, v)$ is close enough to the $D D$ point for the behavior of $A(\mathbf{u})$ to be captured by its linear approximation.

We have shown that the $D D$ points of $2 \times 2$ quasi-linear symmetric systems have, with nonzero probability, a local attracting sector of nonzero width. Therefore, along the simple wave trajectories $\mathbf{u}(\theta)$, the matrix family $A(\mathbf{u}(\theta))$ converges to matrices $A\left(\mathbf{u}^{*}\right)$ with double eigenvalues, unlike the situation in the general case.

\section{Nonsymmetric $2 \times 2$ Case}

In the nonsymmetric case, we are not guaranteed to have a simple wave solution for all matrices $A(\mathbf{u})$ : the eigenvalues can become complex. However, in the domain where simple waves do exist, the dynamics is still homogeneous in $u$ and $v$, and the argument given above for the symmetric case applies.

As above, near a $D D$ point $(0,0)$ one has, to leading order,

$$
\begin{aligned}
A=A_{0}+u A_{u}+v A_{v} & =\left[\begin{array}{ll}
f_{1} & f_{2} \\
f_{3} & f_{4}
\end{array}\right] \\
& =\left[\begin{array}{cc}
\lambda+u a_{1}+v b_{1} & u a_{2}+v b_{2} \\
u a_{3}+v b_{3} & \lambda+u a_{4}+v b_{4}
\end{array}\right] .
\end{aligned}
$$

The system of ODEs that describes the simple waves becomes

$$
\frac{d u}{d v}=\frac{-1}{\frac{f_{1}-f_{4}}{2 f_{2}} \pm \sqrt{\left(\frac{f_{1}-f_{4}}{2 f_{2}}\right)^{2}+\frac{f_{3}}{f_{2}}}} .
$$

Note that

$$
\begin{aligned}
f_{1}-f_{4} & =\left(\lambda+a_{11} u+b_{11} v\right)-\left(\lambda+a_{22} u+b_{22}\right) \\
& =u\left(a_{11}-b_{11}\right)+v\left(a_{22}-b_{22}\right) .
\end{aligned}
$$

Therefore, the system is homogeneous, as in the symmetric case. Introducing again the "angle" $q=\frac{u}{v}$ and picking a "time" $\theta$ such that $d \theta=\frac{d v}{-v}$, the system becomes

$$
\left\{\begin{array}{l}
\frac{d v}{d \theta}=-v \\
\frac{d q}{d \theta}=q-\frac{f_{1}-f_{4}}{2 f_{3}} \pm \sqrt{\left(\frac{f_{1}-f_{4}}{2 f_{3}}\right)^{2}+\frac{f_{2}}{f_{3}}} .
\end{array}\right.
$$

This has the same form as in the symmetric case, so the same argument applies, proving the existence of a sector in phase space that is attracted to the $D D$ point by 


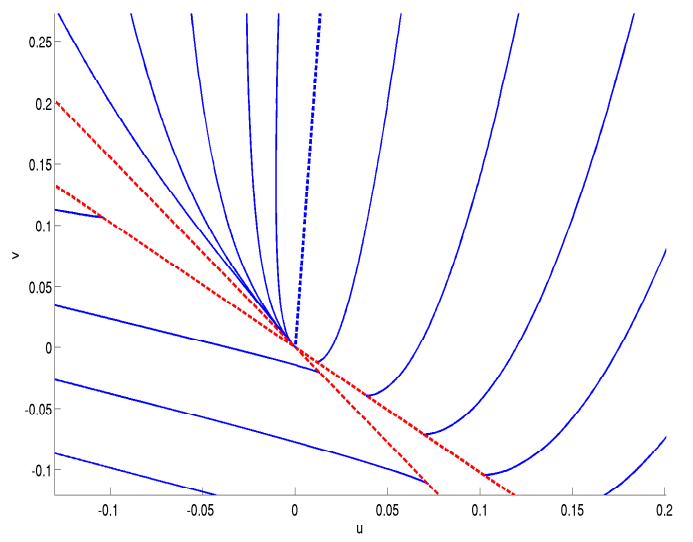

FIGURE 3.1. The $D D$ point $(u, v)=(0,0)$ has an attracting sector on the $(u, v)$ phase plane. The red dashed lines are the boundaries of the elliptic domain. The thick blue dashed line is the boundary of the attracting sector in the hyperbolic domain.

the simple-wave dynamics (see Figure 3.1). We give an example for the following values of parameters:

$$
\begin{aligned}
& A=\left[\begin{array}{ll}
a_{1} & a_{2} \\
a_{3} & a_{4}
\end{array}\right]=\left[\begin{array}{ll}
0.4167 & 0.5962 \\
0.6083 & 1.8114
\end{array}\right], \\
& B=\left[\begin{array}{ll}
b_{1} & b_{2} \\
b_{3} & b_{4}
\end{array}\right]=\left[\begin{array}{ll}
0.8166 & 0.0458 \\
0.5964 & 2.2404
\end{array}\right] .
\end{aligned}
$$

\section{Phase Space Dynamics of Simple Waves in Higher Dimensions}

The $2 \times 2$ case is somewhat special: at $D D$ points, there is no eigenvalue other than the repeated one. It is to be expected, however, that the dynamics of $2 \times 2$ systems near $D D$ points capture the behavior of more general, $n \times n$ systems, with the smooth evolution of the remaining eigenvalues not affecting in a significant way the coalescence of a pair. We show in this section that this is indeed the case, under the assumption that, in the region of phase space that we consider, the matrix $A$ is diagonalizable.

\subsection{Geometric Overview}

In two-dimensional phase space, the $D D$ points are isolated. This is not the situation in dimensions $n$ higher than two, where the $D D$ points generally form a manifold of dimension $n-2$, the $D D$ surface. Hence we need to prove that the phase space dynamics of simple waves carries neighboring points to points on the $D D$ surface, without knowing a priori specifically to which. 
By assumption, $A(w)$ has a complete set of eigenvectors $V_{i}(w)$; we sort them so that the first two correspond to the eigenvalues that coalesce on the $D D$ surface. The simple waves $w(\tau)$ for which our result applies are those corresponding to one of these two families. If we let $W(w)$ stand for either $V_{1}(w)$ or $V_{2}(w)$, the ODE for simple waves becomes

$$
\frac{d w}{d \tau}=W(w) .
$$

Note that $\tau$ is used to denote not the original time in the quasi-linear PDE, but the parameter along a simple wave in $n$-dimensional phase space.

It turns out that near the $D D$ surface, the dynamics parallel to the surface is slow, compared to the fast, two-dimensional, transversal dynamics, which behaves in a fashion entirely analogous to the one for $2 \times 2$ systems. This factorization of the dynamics is best implemented with an appropriate system of coordinates. We first introduce a coordinate system on the $D D$ surface,

$$
s=\left(s_{1}, s_{2}, \ldots, s_{n-2}\right),
$$

so that the vector function $u(s)$ describes the surface in phase space. For any $s$, consider the eigenspace that corresponds to the repeated eigenvalues of $A(u)$ at the point $u(s)$. Since we are free to pick any two linearly independent vectors in it as the first two eigenvectors, we pick $V_{1}(u(s))$ and $V_{2}(u(s))$ so that they are orthonormal and depend smoothly on $s$.

The set of planes spanned by $V_{1}$ and $V_{2}$, parametrized by $s$, foliates phase space in a neighborhood of the $D D$ surface (unless a plane is tangent to the surface, a nongeneric situation that we exclude.) Then we can pick a time-dependent $s$ so that $u(s(\tau))$ shadows the simple wave: for all times $\tau$, the point $w(\tau)$ lies in the plane centered at $u(s(\tau))$ and spanned by the first two eigenvectors of $A(u(s(\tau)))$. Our new coordinates are $\left(x_{1}, x_{2}, s_{1}, \ldots, s_{n-2}\right)$, defining a point in phase space through

$$
w=u(s)+x_{1} V_{1}(u(s))+x_{2} V_{2}(u(s)),
$$

where $x_{1}$ and $x_{2}$ will play the same role as $u$ and $v$ in the two-dimensional case.

The resulting ODE for simple waves in the new coordinate system is a fast-slow dynamical system, where the evolution is slow along the $D D$ surface $u(s)$ and fast in the plane $\left(x_{1}, x_{2}\right)$ transversal to it. The $D D$ surface is a surface of fixed points for this system, with an $n$-dimensional basin of attraction. The rest of this section is devoted to demonstrating this result.

\subsection{Simple Waves in the New Coordinate System $\left(x_{1}, x_{2}, s\right)$}

To analyze the evolution of simple waves, we rewrite (4.1) as

$$
\frac{d}{d \tau}\left[\begin{array}{c}
x_{1} \\
x_{2} \\
s
\end{array}\right]=W,
$$


where the eigenvector $W$ is expressed in the new coordinate system. Not to make the notation cumbersome, from now on we denote by $A$ the matrix $A(w)$ in the new coordinates, which stands for $J^{-1} A(w) J$, where $J$ is the Jacobian matrix of the transformation from $\left(u_{1}, \ldots, u_{n}\right)$ to $\left(x_{1}, x_{2}, s\right)$.

On the $D D$ surface, $\hat{A}=A(u(s))$ is block-triangular,

$$
\widehat{A}=\left[\begin{array}{cc|c}
a & 0 & D \\
0 & a & \\
\hline 0 & B
\end{array}\right],
$$

where $a$ is the repeated eigenvalue.

Note that if the eigenvalues of $\hat{A}$ are $\lambda_{1}, \ldots, \lambda_{n}$, where $\lambda_{1}=\lambda_{2}=a$, then the matrix $B$ has eigenvalues $\lambda_{3}, \ldots, \lambda_{n}$, which we assume to be different from the repeated ones,

$$
\lambda_{i}-a=c_{i}(s) \neq 0, \quad i=3,4, \ldots, n
$$

(in other words, $a$ is a double eigenvalue, not one of higher multiplicity). Then $B$ can be written in the form

$$
B=C+a I,
$$

where $C$ is invertible and has eigenvalues $c_{i}$.

While the simple wave $w(\tau)$ is in a small enough neighborhood of the $D D$ surface, the matrix $A(w)$ is well approximated by $A=\hat{A}+H$, where the elements of $H$ are linear functions of $x_{1}$ and $x_{2}$, with coefficients that depend on $s$. We consider a simple wave that starts in a small enough neighborhood of the $D D$ surface so that at the initial time $\tau=0$ the values of $x_{1}, x_{2}$, and therefore $H$ are of order $\varepsilon \ll 1$. The eigenvalue problem becomes

$$
\left(\left[\begin{array}{cc|c}
\hat{a} & 0 & D \\
0 & \hat{a} & \\
\hline 0 & B-\lambda I
\end{array}\right]+\left[\begin{array}{l|l}
H_{1} & H_{2} \\
\hline H_{3} & H_{4}
\end{array}\right]\right)\left[\begin{array}{l}
\xi_{1} \\
\xi_{2} \\
\xi^{\perp}
\end{array}\right]=0,
$$

where we have written the eigenvector $W$ in the form

$$
W=\left[\begin{array}{l}
\xi_{1} \\
\xi_{2} \\
\xi^{\perp}
\end{array}\right]
$$

where $\xi^{\perp}$ stands for $\left(\xi_{3}, \ldots, \xi_{n}\right)^{\top}$. Because we are interested in the family of simple waves that has a repeated eigenvalue on the $D D$ surface, the difference between $\lambda$ and $a$ is small: $\hat{a}=a-\lambda=O(\varepsilon)$.

Note that under our assumptions the matrix $(B-\lambda I)=C+\widehat{a} I$ is invertible. The last $n-2$ equations in (4.4) are

$$
(C+\hat{a} I) \xi^{\perp}+H_{3}\left[\begin{array}{l}
\xi_{1} \\
\xi_{2}
\end{array}\right]+H_{4} \xi^{\perp}=0 .
$$


Solving this equation to leading order, we obtain

$$
\xi^{\perp}=-C^{-1} H_{3}\left[\begin{array}{l}
\xi_{1} \\
\xi_{2}
\end{array}\right] .
$$

Notice that it follows from this equation that the evolution along the $D D$ surface is slow, since $\xi^{\perp}$ is a higher-order function of $x_{1}$ and $x_{2}$ than $\xi_{1}$ and $\xi_{2}$.

The $n$-dimensional eigenvalue problem (4.4) reduces, to leading order, to the two-dimensional one

$$
\left(\left[\begin{array}{ll}
\hat{a} & 0 \\
0 & \widehat{a}
\end{array}\right]-D C^{-1} H_{3}+H_{1}\right)\left[\begin{array}{l}
\xi_{1} \\
\xi_{2}
\end{array}\right]=0
$$

analogous to the two-dimensional nonsymmetric case of Section 3.

To make the analogy evident, we introduce functions $f_{1}, \ldots, f_{4}$, linear in $x_{1}$ and $x_{2}$ and with coefficients that depend on $s$,

$$
\left[\begin{array}{ll}
f_{1} & f_{2} \\
f_{3} & f_{4}
\end{array}\right]=-D C^{-1} H_{3}+H_{1}
$$

Solving for the eigenvalue $\lambda$, hidden in $\widehat{a}=a-\lambda$, yields

$$
\hat{a}=-\frac{f_{1}+f_{4}}{2} \pm \sqrt{\left(\frac{f_{1}-f_{4}}{2}\right)^{2}+f_{2} f_{3}} .
$$

The eigenvector $\left(\xi_{1}, \ldots, \xi_{n}\right)^{\top}$ is determined by the ratios

$$
\begin{aligned}
& \frac{\xi_{1}}{\xi_{2}}=\frac{1}{f_{3}}\left(\frac{f_{1}-f_{4}}{2} \mp \sqrt{\left(\frac{f_{1}-f_{4}}{2}\right)^{2}+f_{2} f_{3}}\right)=f(q, s), \\
& \frac{\xi^{\perp}}{\xi_{2}}=-C^{-1} H_{3}\left[\begin{array}{c}
\xi_{1} / \xi_{2} \\
1
\end{array}\right] .
\end{aligned}
$$

Notice that the right-hand side of (4.8) depends only on $s$ and the ratio $q=\frac{x_{1}}{x_{2}}$. As in the two-dimensional case, this ratio represents the cotangent of the angle at which the simple wave approaches the $D D$ surface, only now this angle is measured in the moving plane spanned by $V_{1}$ and $V_{2}$, which evolves with the simple wave.

\subsection{Convergence to the $D D$ surface}

Up to now the choice of time $\tau$ along the simple wave has been arbitrary, since the eigenvector $W$ is defined only up to a multiplicative constant. By dividing the equations throughout by $d x_{2} / d \tau$, we eliminate $\tau$ and are ready to introduce the new "time" $\theta$ described below, which illuminates the simple wave convergence to the $D D$ surface. At this stage the ODE for the simple waves has the form

$$
\begin{aligned}
\frac{d x_{1}}{d x_{2}} & =f(q, s), \\
\frac{d s}{d x_{2}} & =-C^{-1} H_{3}\left[\begin{array}{c}
f(q, s) \\
1
\end{array}\right] .
\end{aligned}
$$


To track the convergence of this simple wave to the $D D$ surface $\left(x_{1}, x_{2}\right)=(0,0)$, we pick $\theta$, the time along the simple wave, such that $x_{2}$ converges to 0 as $\theta \rightarrow \infty$, namely $d x_{2} / d \theta=-x_{2}$. The equations for simple waves in the new coordinate system $\left(q, x_{2}, s\right)$ with time $\theta$ become

$$
\begin{aligned}
\frac{d q}{d \theta} & =q-f(q, s), \\
\frac{d x_{2}}{d \theta} & =-x_{2}, \\
\frac{d s}{d \theta} & =x_{2}^{2} C^{-1}(s) H_{3}(q, 1)\left[\begin{array}{c}
f(q, s) \\
1
\end{array}\right] .
\end{aligned}
$$

This is a fast-slow dynamical system, where $x_{2}$ and $q$ are the fast variables, and the $n-2$ components of $s$ are the slow ones. The slow dynamics of $s$ takes place along the $D D$ surface, while the pair $\left(x_{1}, x_{2}\right)$ describes the fast evolution transversal to it. In the new system of coordinates $\left(q, x_{2}, s\right)$, convergence to the $D D$ surface requires $x_{2}$ to stabilize to 0 , and $q$ to converge to some finite value $q^{*}$ (in which case $x_{1}=q x_{2} \rightarrow 0$ as $\theta \rightarrow \infty$ ).

Because $x_{2}$ converges to 0 independently of the rest of the system, the evolution of $s$ from (4.12) is not just slow, but effectively freezes after a time of order $\varepsilon$, implying that $s$ converges to a value $s^{*}$ close to the initial $s(0)$. The equation (4.10) for $q$ becomes a small regular perturbation of the two-dimensional case (3.2): the coefficients now depend on $s$, but are nearly constant, because $s$ is.

We have seen in the two-dimensional nonsymmetric case that $q=f(q, s)$ has an isolated root for some $s_{0}$, which is a stable fixed point for (4.10)-(4.11), with a finite closed domain of attraction. By continuity, this extends to a neighborhood of $s_{0}$, which the simple wave does not leave if it starts close enough to the $D D$ surface. This can be shown by bounding the maximal change in $s$. We assume that the product of all factors but $x_{2}^{2}$ in the right-hand side of (4.12) is bounded by a real positive constant $M$. Integrating (4.12) in $\theta$ from 0 to $\infty$, we obtain that the maximal change of $s$ is less than $\left|x_{2}(0)\right|^{2} M / 2$. We can always choose $x_{2}(0)$ small enough, so that for all $\theta>0, s$ remains in the interval of existence of the isolated stable fixed point of (4.10). As $q$ converges to a finite $q *, x_{2}$ converges to 0 , and so does $x_{1}$. Thus, the simple wave arrives at the $D D$ surface.

\section{Conclusions}

We have shown that the phenomenon of avoidance of eigenvalue crossings, discovered by Wigner and von Neumann for general one-parameter families of matrices, does not apply to the families of matrices associated with the simple waves of nonlinear systems of conservation laws. For these, the loci of double eigenvalues are in fact attractive points in phase space.

Acknowledgments. The authors would like to thank Peter Lax for fruitful discussions, which have considerably improved the clarity of this paper. The work of 
L. Chumakova and E. G. Tabak was partially supported by grants from the National Science Foundation's Division of Mathematics.

\section{Bibliography}

[1] Chumakova, L.; Menzaque, F. E.; Milewski, P. A.; Rosales, R. R.; Tabak, E. G.; Turner, C. V. Shear instability for stratified hydrostatic flows. Comm. Pure Appl. Math. 62 (2009), no. 2, 183197.

[2] Chumakova, L.; Menzaque, F. E.; Milewski, P. A.; Rosales, R. R.; Tabak, E. G.; Turner, C. V. Stability properties and nonlinear mappings of two and three-layer stratified flows. Stud. Appl. Math. 122 (2009), no. 2, 123-137.

[3] Courant, R.; Friedrichs, K. O. Supersonic flow and shock waves. Reprinting of the 1948 original. Applied Mathematical Sciences, 21. Springer, New York-Heidelberg, 1976.

[4] Friedrichs, K. O. Nichtlineare Differenzialgleichungen. Notes of lectures delivered in Göttingen, summer 1955.

[5] Lax, P. D. Hyperbolic systems of conservation laws. II. Comm. Pure Appl. Math. 10 (1957), 537-566.

[6] Lax, P. D. Linear algebra. Pure and Applied Mathematics (New York). Wiley-Interscience, Wiley, New York, 1997.

[7] Milewski, P.; Tabak, E.; Turner, C.; Rosales, R.; Menzaque, F. Nonlinear stability of two-layer flows. Commun. Math. Sci. 2 (2004), no. 3, 427-442.

[8] von Neumann, J.; Wigner, E. Über das Verhalten von Eigenwerten bei adiabatischen Prozessen. Phys. Zschr. 30 (1929), 467-470.

LYUBA CHUMAKOVA

Courant Institute

251 Mercer Street

New York, NY 10012-1185

E-mail: lyubov@cims . nyu.edu
ESTEBAN G. TABAK

Courant Institute

251 Mercer Street

New York, NY 10012-1185

E-mail: tabak@cims.nyu.edu

Received December 2008. 